\title{
STARLINGS HAVE DIFFICULTY IN DETECTING DOT SYMMETRY: IMPLICATIONS FOR STUDYING FLUCTUATING ASYMMETRY
}

\author{
by
}

\begin{abstract}
JOHN P. SWADDLE ${ }^{1)}$ and DOUGLAS A. RUFF ${ }^{2)}$
(Institute for Integrative Bird Behavior Studies, Biology Department, College of William \& Mary, Williamsburg VA 23187-8795, USA)
\end{abstract}

(Acc. 22-IX-2003)

\begin{abstract}
Summary
Small differences between the left and right sides of otherwise symmetric traits are related to developmental instability and can indicate how well the genome is suited to current developmental conditions. As these small asymmetries (termed fluctuating asymmetry, or FA) can reveal how well the genome is suited to the environment, researchers have postulated that FA can indicate fitness and be used by other animals as a visual cue to fitness in sexual and social encounters. Despite these claims, we know little about how animals perceive subtle symmetry differences and whether such cues could be used in nature. Here we investigate the symmetry detection abilities of wild caught European starlings Sturnus vulgaris. We presented symmetric and asymmetric images, that resemble the complex dot patterns commonly observed on starling chest plumage, through a series of operant learning sessions. Asymmetric images were produced by introducing a $40 \%$ dot number asymmetry. Following extended learning sessions, the birds were not able to accurately discriminate symmetry from asymmetry. Although complex dot patterns (similar to those studies here) are common in nature, it appears unlikely that starlings (and perhaps other birds) could use number asymmetry in such traits as a direct cue to mediate behavioural interactions.
\end{abstract}

\section{Introduction}

Fluctuating asymmetries (FAs) are small, random deviations from perfect symmetry that arise during the development of traits that would otherwise

1) Corresponding author's email address: jpswad@wm.edu

2) We are grateful to Dan Cristol, Innes Cuthill, and Paul Heideman for helpful discussion when designing this study. This work was supported by the Thomas F. and Kate Miller Jeffress Memorial Trust and by NSF (IBN-0133795). 
be described as symmetric. These minor asymmetries are related to the ability of the genome to buffer development against environmental perturbations to produce the intended phenotype, i.e. symmetry (Ludwig, 1932; Van Valen, 1962; Parsons, 1990; Clarke, 1993). Recently, FA has received attention because of the seeming relevance of this measure to many areas of biology. For example, FA is known to be affected by genetic factors such as inbreeding and hybridisation (Markow, 1995; Palmer, 1996); FA can increase under unsuitable environmental conditions, such as pollution or over-crowding (Clarke, 1993); also, FA can directly affect performance and fitness through the mechanical disadvantages of asymmetry (Swaddle, 1997; Blackenhorn et al., 1998; Sneddon \& Swaddle, 1999; Bosch \& Marquez, 2000).

As FA can indicate the ability of the genome to cope with current environmental conditions, many biologists have looked for links between asymmetry and general indicators of fitness (reviews in Markow, 1995; Leung \& Forbes, 1996; Palmer, 1996; Clarke, 1998; Lens et al., 2002; Swaddle, 2003). As asymmetry is related to fitness in some species (but by no means all), it has been hypothesized that asymmetry could be used as a direct cue in mate choice and social situations (Møller, 1990). It is also possible that asymmetry could be used as a visual cue in other forms of animal communication, e.g. predator-prey interactions (review in Swaddle, 1999a).

The hypothesis that FA can be used as a direct visual cue (or signal) has generated enormous debate, and there is little consensus among researchers as to the presence or magnitude of a symmetry-signalling effect (Markow, 1995; Leung \& Forbes, 1996; Palmer, 1996; Clarke, 1998; Thornhill \& Møller, 1998; Swaddle, 1999a, 2003). Only a handful of studies have investigated the direct role of small, naturalistic asymmetries in visual communication. A few studies indicate that asymmetry is a visual cue (Swaddle \& Cuthill, 1994; Møller \& Sorci, 1998; Morris \& Casey, 1998); whereas others demonstrate that asymmetry does not affect behavioural interactions (Swaddle \& Witter, 1995; Jablonski \& Matyjasiak, 1997, 2002; Tomkins \& Simmons, 1998). Therefore, there is no clear answer to whether animals use small morphological asymmetries as visual cues in nature. Here we report an experiment that addresses the general issue of whether birds can perceive small asymmetry differences ( $c f$. Swaddle, 1999b).

European starlings Sturnus vulgaris are capable of categorizing symmetry differences in dot patterns, when the asymmetry is produced by a misplacement of dots on one side of the image compared with the other (Swaddle \& 
Pruett-Jones, 2001). Starlings commonly have small white dots at the ends of their dark neck and chest feathers (Feare, 1984) and the number of white dots on a female starling's chest affects social interactions (Swaddle \& Witter, 1995). However, small asymmetries in dot number between left and right sides of the chest (approximately 9\% relative asymmetry) do not appear to affect dominance (Swaddle \& Witter, 1995). The lack of effect of spot asymmetry on social interactions may be due to perceptual limits of birds to detect small dot number asymmetries. Therefore, we tested to see whether starlings can perceive asymmetries in dot numbers where the dot numbers are representative of that observed on starling chest plumage. We hypothesized that starlings will be able to detect large asymmetries, but find it increasingly difficult to detect the small asymmetries that are commonly present in nature (cf. Swaddle, 1999b).

\section{Methods}

Eight wild-caught European starlings (of both sexes) were housed in groups of four in large metal wire cages (approximately $1 \times 0.6 \times 0.7 \mathrm{~m}$ ) with ad libitum chick starter crumbs, drinking water, and bathing water. The birds were maintained on a short day $(8: 16 \mathrm{~h}$ light: dark) photoperiod so they did not undergo gonadal hypertrophy or moult during the experiment (Dawson et al., 1985).

We constructed four operant chambers $(28 \times 26 \times 30 \mathrm{~cm})$. Each chamber consisted of a house light, and two pecking keys linked to a feeder that dispensed a specified volume of chick crumbs into a food trough. Pecking keys were located on either side of the food trough and approximately $10 \mathrm{~cm}$ from the cage floor. The pecking keys were back-illuminated by slide projectors so that we could display slide images on each of the pecking keys. The projected images were approximately $2.5 \times 2.5 \mathrm{~cm}$. We used Coulbourn Instrument's HABITEST apparatus and L2T2 software to design operant schedules to test whether birds could discriminate symmetry from asymmetry in dot stimulus patterns. Birds were trained and tested singly and at no stage in the experiment was there more than one bird in an operant chamber. During all stages of the study, birds were deprived of food for at least $1 \mathrm{~h}$ before being placed in the operant chambers.

Prior to symmetry learning experiments, all birds experienced four pre-exposure sessions and eleven autoshaping sessions. In a pre-exposure session, a small volume of chick crumbs was placed in the food delivery trough and birds were placed in the operant cage for $1 \mathrm{~h}$. All eight birds ate from the trough during this period. In autoshaping trials, pecking keys were illuminated for $10 \mathrm{~s}$, and when the key lights were extinguished a small volume of chick crumbs was delivered to the food trough. There was a $50 \mathrm{~s}$ interval between food delivery and the pecking lights being re-illuminated. During this $50 \mathrm{~s}$ interval, a light inside the food trough was illuminated. This helped to draw the birds' attention to food delivery. An autoshaping session consisted of 20 trials (i.e. 20 min duration). Birds experienced eleven autoshaping sessions (one per day with occasional missed days). All birds ate from the trough and had begun to peck at the keys during these sessions. 
Following the completion of autoshaping sessions, we constructed a protocol in which birds had to peck at illuminated keys to obtain food. When a bird pecked at an illuminated key, a small volume of chick crumbs was delivered in the food trough, the key lights were extinguished, and the food trough light was illuminated for $40 \mathrm{~s}$. After this $40 \mathrm{~s}$ period, the trough light was extinguished and the pecking key lights were re-illuminated (awaiting a further peck from a bird). A pecking session consisted of either 20 pecking trials (as described above) or lasted for $1 \mathrm{~h}$, whichever occurred first. Birds experienced one pecking session per day, with occasional missed days, until all birds had elicited at least 10 trials within a session. As individual birds performed at different levels, some birds experienced more pecking sessions than others (range from four to fourteen). Mean ( $\pm S E M$ ) number of trials elicited during each bird's final pecking session was $14.88( \pm 2.08)$. Birds' pecking performance in these sessions indicated that all eight experimental individuals had learned to associate pecking at illuminated keys with presentation of food. We then progressed to a learning experiment.

The learning experiment examined whether birds could discriminate symmetry from asymmetry where the asymmetry occurred as an unequal number of dots on left and right sides of an image. We constructed 20 pairs of symmetric and asymmetric white dot patterns on a black background using computer graphics software. Within a pair of images, there was an equal number of white (square) dots. Dot number ranged from 50 to 88 (with increments of two dots), which is very similar to the range of dot numbers observed on starling chest and throat plumage (natural range of chest spottiness $=39-92$ ) (Swaddle $\&$ Witter, 1995). Symmetric patterns were generated by taking a random dot pattern (determined by random selection of coordinates for each white dot) with half the number of dots required and reflecting the dot pattern to create two sides of a larger image. Hence, the left side was a mirror reflection of the right. An asymmetric partner for a symmetric image was created by removing dots randomly from one side of the image and placing them in a random position on the opposite side until the pattern had approximately $40 \%$ relative asymmetry. This degree of asymmetry is much larger than commonly observed in starling chest plumage (approximately 9\% (Swaddle \& Witter, 1995)) and was used to maximize the probability that starlings learned to discriminate symmetry from asymmetry. Examples of the dot patterns we used are presented in Fig. 1a, b.

In each operant chamber, a randomly-selected symmetric image (from the set of 20) was displayed on one key (random allocation of left or right key) while a randomly-selected asymmetric image (from the set of 20) was displayed on the other. If the bird pecked at the symmetric image the pecking lights were extinguished, a small volume of food was delivered in the trough, and the trough light was illuminated for $40 \mathrm{~s}$. After $40 \mathrm{~s}$, a new selection of symmetric and asymmetric images was displayed. This completed a learning trial in which a bird pecked at the symmetric image. The 'random' selection of images was restricted so that each individual image could not be shown more than once in a particular session. If the bird pecked at the asymmetric image, the pecking lights and house light were extinguished for $40 \mathrm{~s}$. No food was delivered. Following the $40 \mathrm{~s}$ of darkness, the pecking lights were re-illuminated with a new selection of symmetric and asymmetric images. This completed a learning trial when a bird pecked at an asymmetric image. A learning session consisted of either 20 learning trials or lasted $1 \mathrm{~h}$, whichever occurred first. It is important to stress that all birds were reinforced to peck at symmetric images; hence we expected to see a relative increase in pecking at symmetric images across the learning sessions. We chose to 'train' all birds on symmetric images as data from previous studies indicate that rates of learning 
(a)

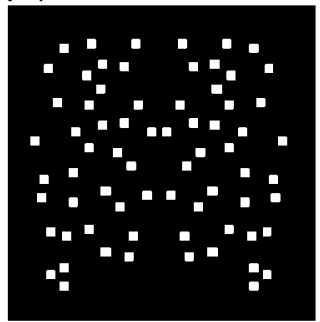

(c)

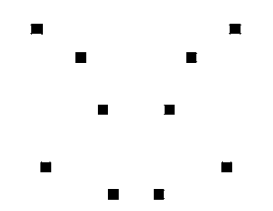

(b)

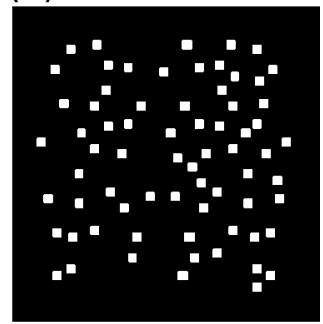

(d)

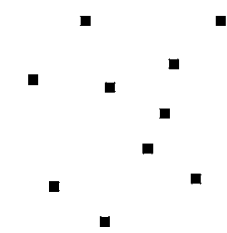

Fig. 1. Examples of the images used in the learning phase. In the dot number experiment, symmetric images (a) possessed mirror symmetry about a vertical axis. Asymmetric images (b) had $40 \%$ fewer dots on one side of the image. In the dot placement experiment (Swaddle $\&$ Pruett-Jones, 2001), the images consisted of dark dots on a light back ground where the symmetric images (c) possessed symmetry in a vertical or horizontal axis. Asymmetric images (d) had an equal number of dots on each side except that dots were not in symmetric positions across the axes.

and overall learning performance are not affected by whether birds are trained on either the symmetric or the asymmetric images (Swaddle, 1999a, b; Swaddle \& Pruett-Jones, 2001). Birds experienced 23 learning sessions (as described previously), one per day with occasional missed days.

Following this initial phase of learning (which the birds were not good at, see Results section), birds were trained with stimuli that we knew starlings could discriminate as symmetric and asymmetric (Swaddle \& Pruett-Jones, 2001). These images were dot images where the asymmetry was produced by moving the position of dots on one side of the image to a position that does not match the dots on the other side (Fig. 1c, d). Following the same overall protocol as before, birds experienced eleven dot position learning sessions, one per day with occasional missed days. It is important to point out that the images used in the previous dot number learning sessions possessed far more elements than the images in the follow-up dot displacement sessions (i.e. Swaddle \& Pruett-Jones, 2001). The overall complexity and number of elements of the dot number images is obviously greater (see Fig. 1).

Following successful completion of the dot position asymmetry sessions, we attempted to re-train the same birds on the original dot number asymmetry stimuli. As the birds had now been exposed to an increased number of learning trials, it is possible that the increased training positively affected their motivation and performance. Therefore, we repeated our initial eleven dot number learning sessions, as described previously.

All data are reported as mean $( \pm S E M)$, unless otherwise stated. All statistical analyses were performed with SPSS (SPSS-Inc., 2000) using two-tailed tests of probability. 


\section{Results}

Surprisingly, the birds showed no evidence of learning to discriminate dot number asymmetry from symmetry (repeated-measures ANOVA: $F_{1,7}=$ $1.34, p=0.29$; Fig. 2a). Relative pecking at the symmetric (correct) key during the last five of the 23 learning sessions was no different than random pecking (paired $t$-test against a null hypothesis of random pecking, $\left.t_{7}=1.15, p=0.29\right)$. The longer-term intention of this experiment was to systematically reduce the relative asymmetry in subsequent learning sessions to explore whether birds exhibit a threshold response to symmetry detection (cf. Swaddle, 1999b).

The lack of discrimination of a $40 \%$ dot number asymmetry could have arisen because this particular group of birds were poorly motivated to feed and, hence, learn in the operant chambers. To test for this directly, in a second experiment we switched learning images to sets of stimuli we knew starlings could discriminate as symmetric and asymmetric (Swaddle \& PruettJones, 2001). As expected, birds displayed a typical asymptotic learning curve (repeated-measures ANOVA: $F_{1,7}=4.82, p=0.064$ ) and showed evidence of being able to discriminate symmetry from asymmetry (performance over last five sessions compared with random pecking, $t_{7}=3.17$, $p=0.016$ ). The birds' performance was comparable with that of Swaddle \& Pruett-Jones' (2001) starlings.

This follow-up experiment showed that our starlings were sufficiently motivated to feed and learn in the operant chambers. Their performance supports the hypothesis that the initial set of images (i.e. dot number asymmetry) posed a particular challenge in terms of symmetry discrimination. To add more weight to this conclusion, we re-examined whether the birds could learn a dot number asymmetry in a subsequent series of learning sessions. There was no evidence that birds could learn a difference between dot number symmetric and asymmetric images when they were switched back to the dot number stimuli for this further series of sessions $\left(F_{1,7}=1.78\right.$, $p=0.224$; Fig. 2b). Mean performance over the last five of these additional sessions was no different to random pecking $\left(t_{7}=1.56, p=0.16\right)$.

In an attempt to help explain why birds could learn symmetry differences in the dot displacement images but not the dot number images, we performed a post-hoc analysis of spatial frequency asymmetries between the two classes of image using Matlab's image processing toolbox. As expected from simple 
(a)

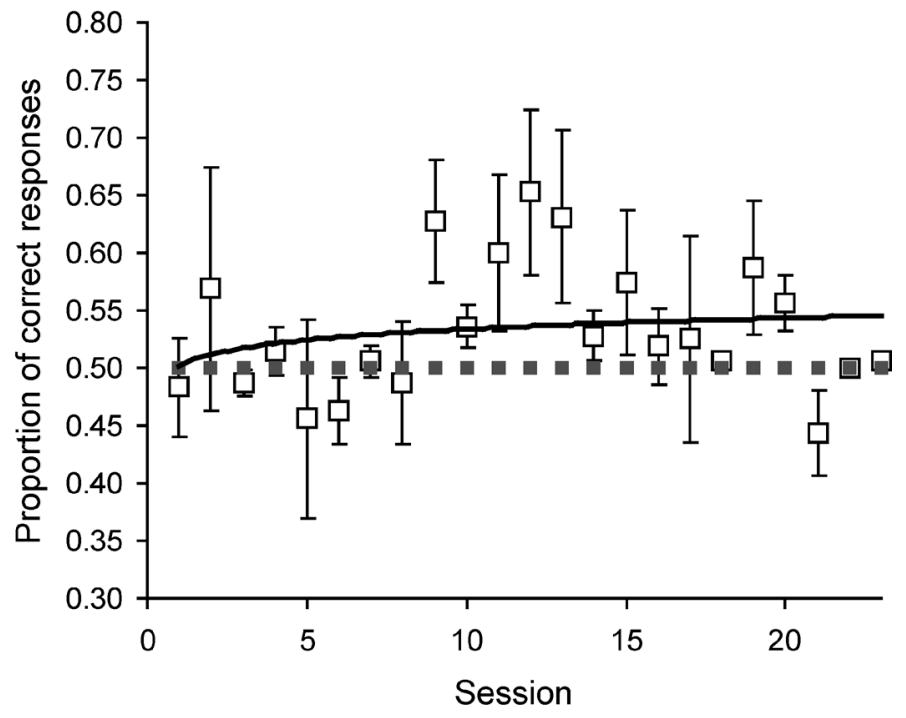

(b)

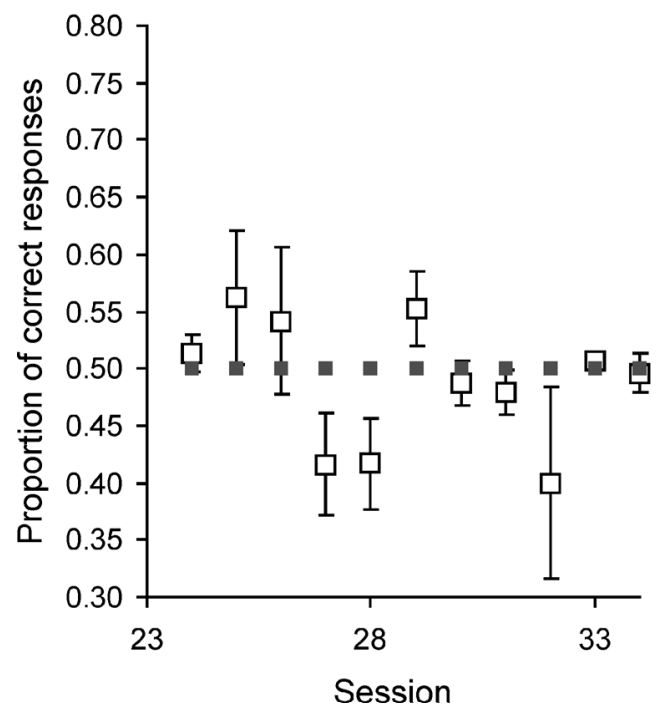

Fig. 2. (a) Mean ( \pm SE) proportion of correct responses (i.e. pecks at symmetric images) when the eight birds were trained with the dot number images. The solid line indicates a logarithmic learning curve $\left(y=0.014 \ln (x)+0.50, r^{2}=0.04\right)$. The dotted line indicates random pecking at left and right keys. (b) Mean $( \pm$ SE) proportion of correct responses (i.e. pecks at symmetric images) when the eight birds were trained with dot number images following their exposure to the dot placement images. 
visual inspection of the images (e.g. Fig. 1), this analysis indicated that the dot displacement asymmetry resulted in a much greater difference in spatial frequency of left versus right sides than the dot number asymmetry.

\section{Discussion}

The birds in our study did not learn a dot number asymmetry task, yet were sufficiently motivated to learn a separate visual operant task under an identical protocol. Therefore, it seems that assessing asymmetry in the form of unequal numbers of dots on left and right side of a pattern is a relatively difficult task, and a symmetry preference is not readily acquired (even following prolonged learning trials).

The images we employed in the dot number task were representative simplifications of starling chest plumage that displayed a much higher degree of dot number asymmetry than often observed in wild birds (Swaddle \& Witter, 1995). If birds cannot detect dot number asymmetry in these exaggerated, yet somewhat naturalistic cues, it seems unlikely that they could reliably discriminate among much more subtle asymmetries in behavioural interactions. Consistent with this conclusion, the social interactions of female starlings is not affected by the symmetric/asymmetric appearance of their chest plumage, yet overall chest dot number positively influences social dominance (Swaddle \& Witter, 1995). The range of asymmetry that Swaddle $\&$ Witter (1995) induced in their experiment was approximately $10 \%$, which is substantially smaller than the $40 \%$ dot number asymmetry employed in our study. Also, we feel that the general form of dot number asymmetry investigated here is likely to be related to developmental instability in starling chest plumage. Asymmetry in the white dots on a starling's chest plumage can easily arise from variation in extent of growth or development, as the white dots are formed at the tips of chest feathers (Feare, 1984).

One important caveat we should raise is that all of our experiments have been performed with flat, two-dimensional images. It is possible that starlings' perception of asymmetry in three-dimensional objects, such as real birds, could be different to those investigated in our study. We intend to extend our studies in the future to include three-dimensional models that are increasingly realistic yet carefully controlled for important visual parameters. 
As dot number asymmetries appear difficult to perceive, it is important to understand how common a dot (or element) number asymmetry task would be in nature. For starlings, it may be very common. These birds possess a spotty plumage throughout much of the year (Feare, 1984; Swaddle \& Witter, 1995). In addition, plumage spots are very common in many avian orders including falcons (Falconiformes), owls (Strigiformes), waders (Charadriformes), quail (Galliformes), and many song birds (Passeriformes). Therefore, plumage spots are a common phenomenon yet our study suggests that it is unlikely that starlings, a common passerine, directly assess dot (or element) number asymmetry.

It is interesting to explore putative explanations for why dot number asymmetry is difficult to assess. We cannot address this question directly from our study, but our data suggest several promising lines for future research. A seemingly crucial component to symmetry detection in biological signals is spatial frequency (i.e. the number of alternating dark and light bands per degree of visual field) (Osorio, 1996; Dakin \& Hess, 1997; Wilkinson et al., 1998; Evans et al., 2000; Rainville \& Kingdom, 2000; Wilson \& Wilkinson, 2002). As remarked earlier, the dot number images possessed greater number of spatial components than the dot displacement images (Fig. 1). If birds view asymmetry as a relative change between left and right sides of an image, altering the overall structure of one side of an image with few elements can dramatically alter the spatial frequency of left versus right, as happened for the dot displacement images. Whereas, making a more subtle removal of elements (i.e. dots) from one side (as in the dot number task) does not dramatically alter the spatial frequency of left compared with right; especially if birds simplify the complex dot number images by grouping elements into contiguous shapes or units, as may happen in human symmetry detection (Jenkins, 1983; Wagemans et al., 1993; Tyler \& Miller, 1994; Wagemans, 1996). Therefore, if starlings are making perceptual judgments by examining spatial frequency changes across an image, we would expect that detecting asymmetry will be easier in the dot displacement than the dot number asymmetry images. Both our theoretical conjecture and our empirical data indicate that complex dot (element) number asymmetries are unlikely visual cues in natural systems. We are currently performing additional experiments to more thoroughly examine our predictions concerning spatial frequency.

An alternative, but unlikely, perception hypothesis is that starlings are making literal correlations or comparisons of individual dot elements between left and right sides. Explicit cross-correlations are difficult to explain 
in human symmetry perception as symmetry can be detected in less than $100 \mathrm{~ms}$ (Dakin \& Hess, 1997). In addition, it seems unlikely that starlings are counting dots on left and right sides when the dot numbers varied from 50 to 88. Although some rats and primates have demonstrated simple arithmetic abilities following extensive training (Dehaene et al., 1998) and swordtail fish Xiphophorus cortezi appear to be able to detect asymmetries among small numbers of stripes on left and right sides of conspecifics (Morris \& Casey, 1998), it would be a big leap to conclude that starlings can count accurately to large numbers and perform some type of subtraction or division.

In general, our study suggests that starlings are not efficient at visualizing asymmetries in element number when the signalling trait is a complex pattern with multiple elements. Potentially, this removes a whole suite of possible cues from the FA-signalling debate and helps us to focus on traits that are relevant to the ways in which birds see their world. Even if asymmetry in plumage dot numbers reveals aspects of fitness, we hypothesize that starlings (and perhaps other birds) are unlikely to use such variation as a direct visual cue as it is appears difficult for potential receivers to discriminate symmetry from asymmetry.

\section{References}

Blackenhorn, W.U., Reusch, T. \& Mühlhäuser, C. (1998). Fluctuating asymmetry, body size and sexual selection in the dung fly Sepsis cynipsea - testing the good genes assumptions and predictions. - J. Evol. Biol. 11, p. 735-753.

Bosch, J. \& Marquez, R. (2000). Typanum fluctuating asymmetry, body size and mate choice in female mid-wife toads (Alytes obstetricans). - Behaviour 137, p. 1211-1222.

Clarke, G.M. (1993). Fluctuating asymmetry of invertebrate populations as a biological indicator of environmental quality. - Environ. Poll. 82, p. 207-211.

- - (1998). Developmental stability and fitness: the evidence is not quite so clear. - Am. Nat. 152 , p. $762-766$.

Dakin, S.C. \& Hess, R.F. (1997). The spatial mechanisms mediating symmetry perception. — Vis. Res. 37, p. 2915-2930.

Dawson, A., Goldsmith, A.R. \& Nicholls, T.J. (1985). Thyroidectomy results in termination of photorefractoriness in starlings (Sturnus vulgaris) kept in long day lengths. - J. Reprod. Fert. 74, p. 527-533.

Dehaene, S., Dehaene-Lambertz, G. \& Cohen, L. (1998). Abstract representations of numbers in the animal and human brain. - Trends Ecol. Evol. 21, p. 355-361.

Evans, C.S., Wenderoth, P. \& Cheng, K. (2000). Detection of bilateral symmetry in complex biological images. - Perception 29, p. 31-42.

Feare, C. (1984). The starling. — Oxford University Press, Oxford. 
Jablonski, P.G. \& Matyjasiak, P. (1997). Chaffinch (Fringilla coelebs) epaulette display depends on the degree of exposure but not symmetry of intruder's epaulettes. - Behaviour 134, p. 1115-1121.

- — \& - - (2002). Male wing-patch asymmetry and aggressive response to intruders in the common chaffinch (Fringilla coelebs). - Auk 119, p. 566-572.

Jenkins, B. (1983). Component processes in the perception of bilaterally symmetric dot textures. - Perc. Psychophys. 34, p. 433-440.

Lens, L., Van Dongen, S., Kark, S. \& Matthysen, E. (2002). Fluctuating asymmetry as an indicator of fitness: can we bridge the gap between studies? - Biol. Rev. 77, p. 27-38.

Leung, B. \& Forbes, M.R. (1996). Fluctuating asymmetry in relation to stress and fitness: Effects of trait type as revealed by meta-analysis. - Ecoscience 3, p. 400-413.

Ludwig, W. (1932). Das Rechts-Links Problem im Tierreich und beim Menschen. Springer-Verlag, Berlin.

Markow, T.A. (1995). Evolutionary ecology of developmental instability. - Ann. Rev. Entomol. 40, p. 105-120.

Møller, A.P. (1990). Fluctuating asymmetry in male sexual ornaments may reliably reveal male quality. - Anim. Behav. 40, p. 1185-1187.

— — \& Sorci, G. (1998). Insect preference for symmetrical artificial flowers. — Oecologia 114, p. 37-42.

Morris, M.R. \& Casey, K. (1998). Female swordtail fish prefer symmetrical sexual signal. — Anim. Behav. 55, p. 33-39.

Osorio, D. (1996). Symmetry detection by categorization of spatial phase, a model. — Proc. R. Soc. Lond. B 263, p. 105-110.

Palmer, A.R. (1996). Waltzing with asymmetry. - Bioscience 46, p. 518-532.

Parsons, P.A. (1990). Fluctuating asymmetry: An epigenetic measure of stress. — Biol. Rev. 65, p. 131-145.

Rainville, S.J.M. \& Kingdom, F.A.A. (2000). The functional role of oriented spatial filters in the perception of mirror symmetry - Psychophysics and modeling. - Vis. Res. 40, p. 2621-2644.

Sneddon, L.U. \& Swaddle, J.P. (1999). Asymmetry and fighting performance in the shore crab, Carcinus maenas. - Anim. Behav. 58, p. 431-435.

SPSS-Inc. (2000). SPSS for Windows user's guide. - SPSS Inc., Chicago.

Swaddle, J.P. (1997). Within-individual changes in developmental stability affect flight performance. - Behav. Ecol. 8, p. 601-604.

— - (1999a). Is fluctuating asymmetry a visual signal? — In: Animal signals (Y. Espmark, T. Amundsen \& G. Rosenqvist, eds). Tapir Academic Press, Trondheim, p. 155-175.

_ — (1999b). Limits to length asymmetry detection in starlings: implications for biological signalling. - Proc. R. Soc. Lond. B 266, p. 1299-1303.

— - (2003). Fluctuating asymmetry, animal behavior, and evolution. — Adv. Anim. Behav. in press.

— — \& Cuthill, I.C. (1994). Female zebra finches prefer males with symmetric chest plumage. - Proc. R. Soc. Lond. B 258, p. 267-271.

- — \& Pruett-Jones, S. (2001). Experimental investigation of the acquisition of learned symmetry discrimination in starlings. - Am. Nat. 158, p. 300-307.

— — \& Witter, M.S. (1995). Chest plumage, dominance and fluctuating asymmetry in female starlings. - Proc. R. Soc. Lond. B 260, p. 219-223. 
Thornhill, R. \& Møller, A.P. (1998). The relative importance of size and asymmetry in sexual selection. - Behav. Ecol. 9, p. 546-551.

Tomkins, J.L. \& Simmons, L.W. (1998). Female choice and manipulations of forceps size and symmetry in the earwig Forficula auricularia L. - Anim. Behav. 56, p. 347-356.

Tyler, C.W. \& Miller, R.H. (1994). Pattern identification by trajectory analysis in autocorrelation hyperspace. - Proc. World Congress Neural Networks 3, p. 312-316.

Van Valen, L. (1962). A study of fluctuating asymmetry. - Evolution 16, p. 125-162.

Wagemans, J. (1996). Detection of visual symmetries. — In: Human symmetry perception and its computational analysis (C.W. Tyler, ed.). VSP, Utrecht, p. 25-48.

_ _, Van Gool, L., Swinnen, V. \& Van Horebeek, J. (1993). Higher-order structure in regularity detection. - Vis. Res. 33, p. 1067-1088.

Wilkinson, F., Wilson, H.R. \& Habak, C. (1998). Detection and recognition of radial frequency patterns. - Vis. Res. 38, p. 3555-3568.

Wilson, H.R. \& Wilkinson, F. (2002). Symmetry perception: a novel approach for biological shapes. - Vis. Res. 42, p. 589-597. 